\title{
THE INFLUENCE OF ATOMS-IN-MOLECULES METHODS ON SHARED-ELECTRON DISTRIBUTION INDICES AND DOMAIN AVERAGED FERMI HOLES
}

\author{
Patrick Bultinck ${ }^{*}, 1$, David L. Cooper², Robert Ponec ${ }^{3}$ \\ 1. Department of Inorganic and Physical Chemistry, Ghent University, Krijgslaan 281 (S3), 9000 \\ Gent, Belgium. Patrick.Bultinck@UGent.be \\ 2. Department of Chemistry, University of Liverpool, Liverpool, L69 7ZD, UK \\ 3. Institute of Chemical Process Fundamentals, Academy of Sciences of the Czech Republic \\ v.v.i., Prague 6, Suchdol 2, 165 02, Czech Republic.
}

The effect of using different methods to obtain Atoms in Molecules (AIM) on the Shared-Electron Distribution Indices (SEDI) and Domain Averaged Fermi Holes (DAFH) is examined using a test set of diatomic molecules. Use of Bader's binary AIM model gives significantly different SEDI as a function of internuclear distances than do self consistent Hirshfeld based AIM models. DAFH eigenvectors remain very similar for all AIM among the different methods. The corresponding eigenvalues are found to differ significantly although the sums of complementary eigenvalues show only relatively small changes. The choice of a binary division of molecular space into AIM domains is found to lead to extra structure in SEDI curves, but this probably has limited chemical significance.

P. Bultinck, D.L. Cooper, R. Ponec, J. Phys. Chem. A, Accepted for publication, Special issue for Klaus Ruedenberg, (2010). 